\title{
Un programa de manejo basado en la familia resultó beneficioso en niños obesos
}

\author{
A family-based program was effective in obese children
}

\section{Objetivo}

Comparar con la atención convencional, los efectos de un programa intensivo basado en la familia (PIBF) para el manejo de la obesidad infantil.

\section{Diseño, pacientes y lugar}

Ensayo clínico aleatorizado y controlado de un año de seguimiento sobre 209 niños de 8 a 16 años de edad con un percentilo de índice de masa corporal (IMC) mayor a 95 para edad y sexo. 135 fueron seguidos durante seis meses y 119 durante 12. Clínica de obesidad pediátrica de la Universidad de Yale, EEUU.

\section{Intervención}

Grupo control $(n=69)$ : tratamiento convencional y consejería semestral. Grupo PIBF $(n=105)$ : dos consultas semanales durante los primeros seis meses y luego, quincenales. Ver cuadro 1.

Cuadro 1: componentes del programa intensivo.

- Educación nutricional para niños y padres (son quienes van de compras) sobre elecciones alimentarias y tamaño de las porciones, desalentando las "dietas" ya que sólo promueven resultados de corto plazo.

- Dos sesiones semanales de 50 minutos de actividad física moderada (varios deportes y videos interactivos de baile) promoviendo que los participantes realicen tres sesiones semanales más por su cuenta.

- Utilizando películas y libros didácticos, dietistas y trabajadores sociales trabajan con grupos de niños (sin sus padres presentes) en talleres de 40 minutos con el objetivo de que reconozcan situaciones gatilladoras de "sobre-ingesta" y aprendan a manejarlas. Por separado trabajan con los padres para que aprendan a ser modelos de rol y a promover cambios saludables en el entorno familiar.

Resumido de: www.brightbodies.org
Savoys M y col. JAMA 2007;297:2697-2704

\section{Medición de resultados principales}

Cambio en el peso corporal, en el IMC, en la grasa corporal y en el modelo de homeostasis de resistencia a la insulina a los seis y a los 12 meses.

\section{Resultados Principales}

El grupo PIBF presentó una mejoría sostenida a los 12 meses se resume en la tabla 1 .

Tabla 1: efectos metabólicos al año del programa de manejo de peso.

\begin{tabular}{l|c|c}
\hline \multirow{2}{*}{ Aumento de peso $\mathbf{( k g )}$} & \multicolumn{2}{|c}{ Resultado de la intervención (IC95\%) } \\
\cline { 2 - 3 } & $\begin{array}{c}\text { Intensiva y basada } \\
\text { en la familia }\end{array}$ & Convencional \\
\hline Variación en el índice de masa corporal $\mathbf{( k g / \mathbf { m } ^ { 2 } )}$ & $0,3(-1,4$ a 2$)$ & $7,7(5,3$ a 10$)$ \\
\hline Porcentaje de la grasa corporal $\mathbf{( \% )}$ & $-1,7(-2,3$ a $-2,1)$ & $1,6(0,8-2,3)$ \\
\hline Resistencia a la insulina (HOMA-IR) & $-3,7(-5,4$ a $-2,1)$ & $5,5(3,2$ a 7,8$)$ \\
\hline
\end{tabular}

HOMA IR: resulta de multiplicar la insulinemia en ayunas $(\mathrm{uU} / \mathrm{mL})$ por la glucemia basal ( $\mathrm{mg} / \mathrm{dL}$ ) dividiendo el resultado por 405 (una constante). Valores superiores a 2,5 implican insulinorresistencia.

\section{Conclusión}

Un PIBF para el manejo de peso resultó beneficioso para el tratamiento de niños obesos.

Palabras Claves: Obesidad infantil, programas, síndrome metabólico. Key Words: childhood obesity, programs, metabolic syndrome. Fuente de financiamiento: National Institute Health y Fundación McPhee Bristol, EE.UU.

\section{Comentario}

La obesidad es un problema crónico prevalente en la consulta ambulatoria pediátrica con malos resultados con el tratamiento convencional. Requiere cambios rotundos en el estilo de vida y debe incluir a los cuidadores para que tenga éxito.

Este estudio demuestra que un PIBF tiene mayor impacto que la intervención tradicional, confirmando los resultados de un estudio previo no aleatorizado con dos años de seguimiento'. Las estrategias que apuntan a modificar hábitos logran mayor éxito que las dietas estructuradas, debido a la baja adherencia a estas últimas, como se evidenció con una rama discontinuada en este estudio y otros estudios ${ }^{2-3}$.

Los resultados al año son alentadores, habiendose logrado neutralizar el aumento de peso y disminuir el IMC gracias a un aumento de la talla durante ese tiempo. A la disminución prome- dio de 1,7 puntos de IMC -lo que ya es un logro- debemos sumarle el promedio de 1,6 puntos que subió el grupo control, lo que implica una diferencia total de 3,3 puntos. La disminución de la resistencia insulínica sería protectora en el largo plazo ya que está implicada en la fisiopatogenia de la obesidad y sus complicaciones ${ }^{4}$.

\section{Conclusiones del comentador}

Un PIBF ayuda a frenar el aumento de peso y mejorar parámetros metabólicos en niños obesos al año de seguimiento. Quedan como interrogantes si su complejidad y sus costos permiten implementarlo en la práctica cotidiana.

Julio Busaniche [ Servicio de Clínica Pediátrica del Departamento de Pediatría del Hospital Italiano de Buenos Aires. julio.busaniche@hospitalitaliano.org.ar ]

Busaniche J. Un programa de manejo basado en la familia resultó beneficioso en niños obesos. Evid. actual. práct. ambul; 11 (3):73, May-Jun.2008. Comentado de: Savoye M y col. Effects of a Weight Management Program on Body Composition and metabolic Parameters in Overweight Children. A Randomized Controlled Trial. JAMA 2007;297:2697- 2704. PMID: 17595270.

\section{Referencia}

1. Savoye M, y col. Anthropometric and psycosocial changes in obese adolescents enrolled in a weigth management program. J Am Diet Assoc. 2005; 105:364-370.

2. Field A y col. Relation between dieting and weight change among preadolescents and adolescents. Pediatrics. 2003; 112:900-906.

3. Neumark-Sztainer D y col. Obesity, disordered eating, and eating disorders in a longitudinal study of adolescents: how do dieters fare 5 years later? $\mathrm{J}$ Am Diet Assoc. 2006;106:559-568.

4. Caprio S. Insulin resistence in childhood obesity. J Pediatric Endocrinol Metab. 2002;15(suppl 1):487-492. 